\title{
PREMIÈRE CARACTÉRISATION MORPHOLOGIQUE, BIOLOGIQUE ET GÉNÉTIQUE DES POPULATIONS DE GRANDE ALOSE (ALOSA ALOSA) ET D'ALOSE FEINTE (ALOSA FALLAX SPP.) \\ DE LA CHARENTE (FRANCE).
}

\author{
V. VÉRON (1, 2), R. SABATIÉ (1,2), J.L. BAGLINIÈRE (2), P. ALEXANDRINO (3)
}

(1) Laboratoire d'Halieutique, ENSAR, 65 rue de Saint Brieuc, 35042 RENNES Cedex, France. E-mail : veron@epi.roazhon.inra.fr

(2) UMR INRA-ENSA Ecobiologie et Qualité des Hydrosystèmes Continentaux, 65 rue de Saint Brieuc, 35042 RENNES Cedex, France.

(3) CECA ICETA, Université de Porto Campus de Agrario, R. Monte Crasto, 4480 VILA DO CONDE, Portugal.

\section{RÉSUMÉ}

Les deux espèces d'aloses atlantiques (Alosa alosa et $A$. fallax) colonisent la Charente mais leurs populations n'ont jamais été décrites à la différence de celles des grands fleuves français voisins. Les propriétés physiques du bassin sont présentées en terme de qualité de l'eau, d'habitats et d'obstacles à la migration. Les grandes aloses colonisent la Charente jusqu'en amont d'Angoulême $(171 \mathrm{~km})$ lorsque les conditions hydrauliques des mois d'avril et mai effacent les 21 barrages situés en aval. Des frayères actives ont été localisées sur cette portion du fleuve. Les aloses feintes sont bloquées plus en aval par le barrage de Crouin (102 km, aval de Cognac) et une seule frayère active a été repérée. L'abondance de ces deux stocks d'alose ne peut pas être estimée car les seules données disponibles, faute de dispositifs de contrôle des migrations, proviennent des captures faites par pêche professionnelle et à la ligne.

La caractérisation morphologique et génétique des deux espèces a été faite à partir de comptages méristiques, de mesures morphométriques et de prélèvements (écaille, tissus et sang) réalisés en 1997 et 1998. Le nombre total moyen de branchiospines du premier arc branchial gauche chez les grandes aloses est de 125 contre 42 chez les feintes. Quelle que soit l'espèce et à âge égal, les femelles ont une taille moyenne supérieure à celle des mâles. Les femelles de grande alose sont âgées en moyenne de 5 ans et les mâles de 4 ans, aucun des poissons échantillonnés ne présentant de marques de reproduction antérieure. Les femelles et les mâles d'alose feinte sont âgés en moyenne de 3 ans, $12,5 \%$ des femelles se sont déjà reproduites une fois et 3,5\% deux fois. Chez les mâles, $40 \%$ se sont déjà reproduits une fois. La plage temporelle de reproduction a été déterminée par suivi de terrain et enquête. Celle des grandes aloses se situe entre la première quinzaine de mai et la première quinzaine de juin. Celle des aloses feintes au cours de la seconde quinzaine de mai et la première quinzaine de juin. La comparaison de leurs caractéristiques à celle des populations déjà étudiées montre qu'elles restent assez proches des stocks ligériens. 
La caractérisation génétique des deux stocks a été effectuée par électrophorèse des protéines (8 locus). Celle des grandes aloses de la Charente présente des distributions de fréquences alléliques relativement proches de celles des populations caractérisées antérieurement mais se distingue par des taux d'hétérozygotie plus faible et par un plus grand nombre de locus hétérozygotes. Globalement, les aloses feintes de la Charente apparaissent beaucoup moins polymorphes que celles de l'ensemble des populations atlantiques étudiées. La comparaison globale des populations françaises, portugaises et marocaines par l'analyse des distances génétiques confirme le caractère très peu polymorphe des populations de grande alose par rapport à celles d'alose feinte.

Mots-clés: Alosa alosa, Alosa fallax, Charente, France, morphologie, biologie, écologie, génétique.

\section{FIRST MORPHOLOGICAL, BIOLOGICAL AND GENETIC CHARACTERISTICS OF SHADS (ALOSA ALOSA AND ALOSA FALLAX SPP) IN THE RIVER CHARENTE (FRANCE).}

\section{ABSTRACT}

Two species of Atlantic shads (Alosa alosa and Alosa fallax) colonize Charente but their populations have never been described contrary to those of the largest French rivers. The physical properties of the basin are presented: quality of water, habitats and obstacles to the migration. The allis shad colonize Charente till Angouleme $(171 \mathrm{~km})$ when the hydraulic conditions of May and April erase the 21 dams located downstream. Active spawning areas were located. Twaite shads are blocked downstream by the dam Crouin (102 km Cognac downstream) and only one active spawning area was located. The abundance of these two stocks of shads cannot be estimated because the only data available are captures made by the commercial fishing and angling.

The morphological and genetic characterization of the two species was made by counting meristic, morphometric measurements and taking away (scale, muscle tissue and blood) carried out in 1997 and 1998. The average total number of branchiospines of the first left branchial arch for the allis shad is 125 against 42 for the twaite shads. The relation between length and weight was calculated for each species and according to the date of capture. Whatever the species, the females have an average size higher than males. The females of allis shad are old on average 5 years and males 4 years old, none sampled fish presented marks of previous reproduction. The females and the males of twaite shads are old on average 3 years, $12.5 \%$ of the females already reproduced once and $3.5 \%$ twice. In the males, $40 \%$ already reproduced once. The temporal range of reproduction was determined by follow-up of ground and investigations. That of the allis shad is located between the first fortnight of May and the first fortnight of June. That of the twaite shads during the second fortnight of May and the first fortnight of June. The comparison of their characteristics with the one of shads coming from stocks already studied indicates that they are closed to the populations of shads coming from the Loire river.

The genetic characterization of two stocks was carried out by electrophoresis of the proteins (8 loci). That of allis shad of Charente presents some allelic frequencies distributions relatively close to those of the populations characterized before but is characterized by lower rates of heterozygoty and by a greater number of heterozygous loci. All in all, the twaite shads of Charente appear much less polymorph than those of the whole of the studied Atlantic populations. The comparison of the French, Portuguese and 
Moroccan populations by the analysis of the genetic distances confirms the character far from polymorph of the populations of allis shad compared to those of twaite shad.

Key-words : Alosa alosa, Alosa fallax, Charente, France, morphology, biology, ecology, genetics.

\section{INTRODUCTION}

Les aloses atlantiques (Alosa alosa et Alosa fallax spp.) sont considérées comme des espèces vulnérables au niveau européen (critères de l'IUCN), en raison principalement de la réduction drastique de leur aire de répartition et de la qualité de leurs habitats en eau douce. Ainsi, elles sont inscrites sur le livre rouge des espèces menacées en Espagne, France et Portugal. De plus, elles figurent à l'annexe III de la convention de Berne (exploitation réglementée) et aux annexes II et $\mathrm{V}$ de la Directive Habitat Faune-Flore (zones spéciales de conservation) (BAGLINIERE et ELIE, 2000). De fait, la gestion, la sauvegarde et la protection de ces espèces patrimoniales passent par une connaissance précise des populations encore en place et donc par une identification de leurs caractéristiques biologiques et génétiques ainsi qu'une évaluation de leur niveau d'abondance.

Jusqu'à présent, les études concernant la biologie des populations et l'état des stocks des deux espèces d'aloses présentes (Alosa alosa et Alosa fallax) ont été menées sur les grands fleuves encore colonisés en France : Loire (MENESSON-BOISNEAU et BOISNEAU, 1990) ; Garonne (CASSOU-LEINS et CASSOU-LEINS, 1981 ) ; Gironde (TAVERNY, 1991; ROCHARD, 1992); Adour (PROUZET et al., 1994); Rhône (LE CORRE, 1999). II en a été de même pour les études concernant l'identification génétique des espèces et de leurs hybrides qui ont d'abord été réalisées sur la Loire (BOISNEAU et al., 1992) avant d'être reprises au Portugal (ALEXANDRINO, 1996). Aucune étude n'avait donc été entreprise jusqu'à présent sur les populations d'aloses des petits fleuves côtiers de la façade Manche Atlantique française, alors que certains d'entre eux semblent colonisés par les aloses et possèdent des zones de reproduction fonctionnelles pour ces espèces.

Ainsi, la présente étude s'est intéressée aux populations des deux espèces d'aloses qui colonisent la Charente et qui n'avaient jamais été étudiées jusqu'à présent. L'objectif de ce travail était de compléter les connaissances écobiologiques et génétiques des populations, acquises sur l'aire de répartition des deux espèces. Après une présentation du cadre de l'étude, les résultats sont présentés et discutés en analysant la zone de colonisation, les structures biométriques, démographiques et génétiques des populations et en les replaçant dans un cadre biogéographique élargi.

\section{MATÉRIEL ET MÉTHODES}

\section{Zone d'étude}

D'une surface de $10100 \mathrm{~km}^{2}$, le bassin versant de la Charente se situe entre celui de la Garonne et celui de la Loire (Figure 1). D'une longueur de $360 \mathrm{~km}$, son débit moyen à Jarnac est de $48 \mathrm{~m}^{3} / \mathrm{s}$ avec une valeur de débit minimale de $5 \mathrm{~m}^{3} / \mathrm{s}$ et maximale de $400 \mathrm{~m}^{3} / \mathrm{s}$ (données Agence de l'Eau Adour-Garonne, 1996) ce qui en fait un fleuve au régime relativement calme. Le substrat calcaire de ce bassin rend ses eaux exceptionnellement claires et permet des observations visuelles des poissons. La marée dynamique se fait ressentir jusqu'à la Baine (amont de Saintes). 
La qualité de l'eau reste passable (60\% des points de mesure, 1992-1993, normes Agence de l'Eau), voire médiocre (20\%), sur de nombreux cours d'eau du bassin. Une amélioration de la qualité de l'eau est constatée sur les petits tributaires (Son-Sonnette, Antenne, Couture et Bonnieure), une détérioration est constatée sur la Charente amont et la Charente en aval de Saintes. Les paramètres déclassant pour l'ensemble du cours de la Charente sont l'ammoniaque, les matières oxydables qui provoquent des carences en oxygène dissous à l'aval de Cognac, mais aussi les nitrates et le phosphore.

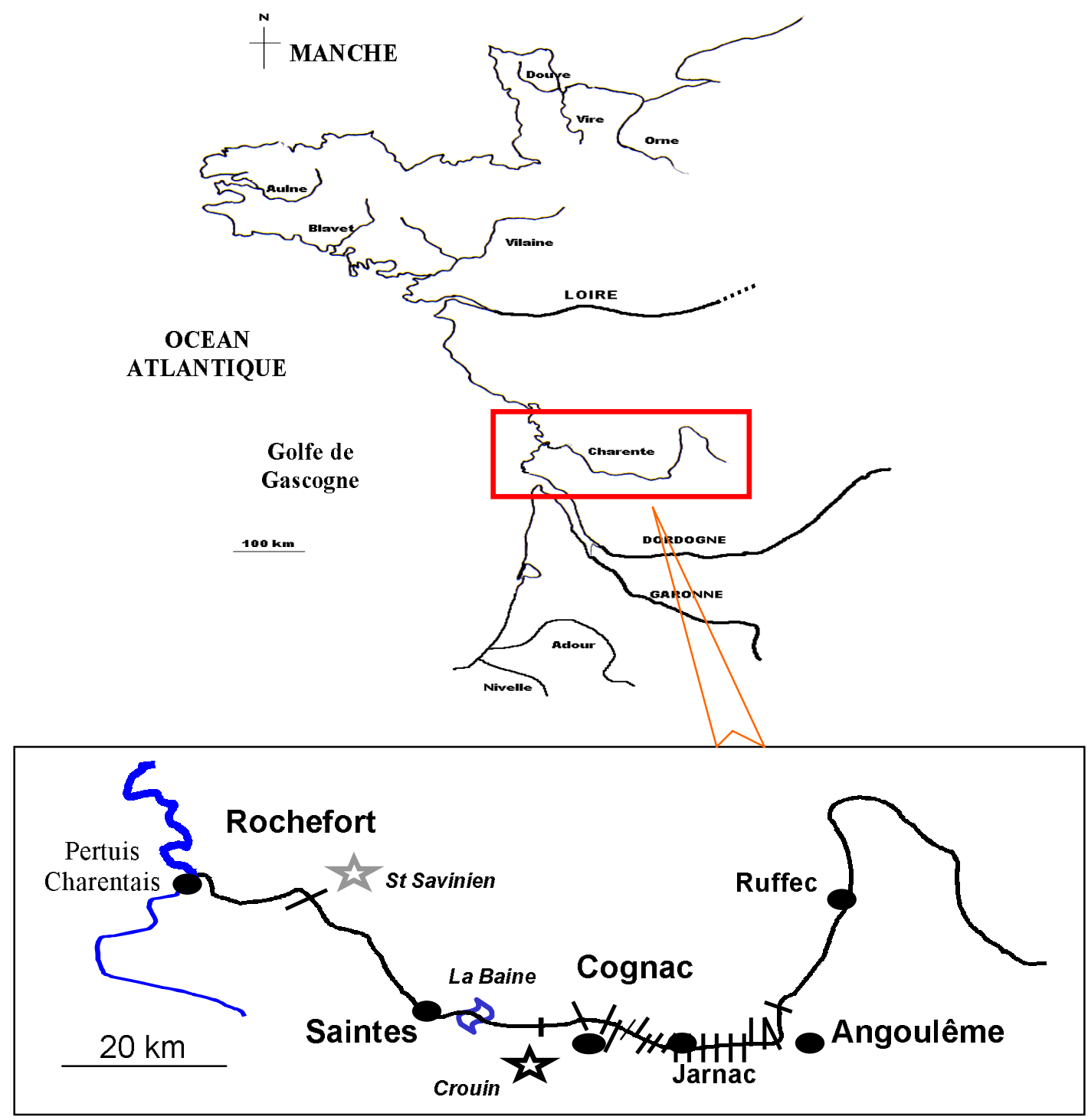

Figure 1

Obstacles (-) à la migration des aloses sur la Charente et secteurs d'échantillonnage. Etoile grise : échantillonnage des aloses feintes ; étoile noire : échantillonnage des grandes aloses ; vague : limite de la marée dynamique

Figure 1

Obstacles (-) to the migration of shads in the river Charente and sampling area. Grey star : sampling of twaite shad; black star : sampling of allis shad ; wave : limit of dynamic tide. 


\section{Barrages, zones de reproduction et front de migration}

Les barrages de navigation, obstacles à la migration des aloses sur le bassin de la Charente sont nombreux, celle ci étant navigable jusqu'à l'écluse de Saint Cybard, à l'amont d'Angoulême. Ainsi, de l'estuaire à Angoulême, il existe 21 écluses sur la Charente. Ces barrages sont soit des vannes déversantes (comme par exemple à Bourg Charente), soit des vannes de fond de type wagon (comme par exemple à Garde-Moulin), soit des clapets déversant et une vanne de type wagon (le plus fréquent sur la Charente), comme par exemple à Crouin. II n'existe aucun moyen technique de contrôle des migrations d'aloses sur le bassin de la Charente, le front de migration a donc été déterminé par observations visuelles des bans de poissons en aval des ouvrages et par enquête halieutique. Les frayères actives ont été repérées de nuit par observations visuelles et sonores.

\section{Caractéristiques biologiques}

\section{Techniques d'échantillonnage}

60 grandes aloses et 34 aloses feintes ont été échantillonnées en 1997 et 1998 par la Cellule Mlgrateur (CMI) du Conseil Supérieur de la Pêche de Poitiers et par un pêcheur professionnel au niveau de Saint Savinien (Tableau I et Figure 1).

\section{Tableau I}

Techniques de captures et Nombre d'aloses échantillonnées (N). (Aa : grande Alose ; Af : Alose feinte).

\section{Table I}

Catch technics and number of shads sampled (N).

\begin{tabular}{|c|c|c|c|c|c|c|}
\hline Année & Date & $\mathbf{N}$ & Esp & Mode de capture & lieu & Pêcheur \\
\hline 1997 & $\begin{array}{l}6 \text { mai } \\
10 \text { et } 16 \text { juin }\end{array}$ & 57 & $\mathbf{A a}$ & $\begin{array}{l}\text { filet maillant (longueur : } 25 \mathrm{~m} \text {, } \\
\text { hauteur : } 3 \mathrm{~m} \text {, maille : } 60 \mathrm{~mm} \text { de coté) }\end{array}$ & $\begin{array}{l}\text { Barrage de } \\
\text { Crouin }\end{array}$ & CMI (CSP) \\
\hline 1997 & 6 mai & 1 & Af & $\begin{array}{l}\text { filet maillant (longueur : } 25 \mathrm{~m} \text {, } \\
\text { hauteur : } 3 \mathrm{~m} \text {, maille : } 60 \mathrm{~mm} \text { de coté) }\end{array}$ & $\begin{array}{l}\text { Barrage de } \\
\text { Crouin }\end{array}$ & CMI (CSP) \\
\hline 1998 & 13 mai & 3 & $\mathbf{A a}$ & $\begin{array}{l}\text { filet maillant (longueur : } 25 \mathrm{~m} \text {, } \\
\text { hauteur : } 3 \mathrm{~m} \text {, maille : } 60 \mathrm{~mm} \text { de coté) }\end{array}$ & $\begin{array}{l}\text { Barrage de } \\
\text { Crouin }\end{array}$ & CMI (CSP) \\
\hline 1998 & mai & 33 & Af & $\begin{array}{l}\text { filet maillant (longueur : } 40 \mathrm{~m} \text {, } \\
\text { hauteur : } 3 \mathrm{~m} \text {, maille : } 60 \mathrm{~mm} \text { de coté) }\end{array}$ & $\begin{array}{l}\text { Barrage de } \\
\text { St Savinien }\end{array}$ & $\begin{array}{l}\text { Pêcheur } \\
\text { professionnel }\end{array}$ \\
\hline
\end{tabular}

\section{Récolte des données}

Tous les poissons capturés ont été mesurés et pesés. A cela se sont ajoutées les mesures de différentes variables biométriques et méristiques habituellement utilisées pour les aloses (MENNESSON-BOISNEAU et BOISNEAU, 1990 ; TAVERNY, 1991 ; SABATIE, 1993 ; SABATIE et al., 2000). Par ailleurs, des écailles ont été prélevées sur tous les individus en tenant compte des recommandations de MENNESSON-BOISNEAU et BAGLINIERE (1992). 


\section{Traitement des données}

La distribution des données biométriques et méristiques a été étudiée par espèce. Les relations entre les deux variables Lt (longueur totale) et Lf (longueur fourche), d'une part, et Lt et PDS év. (poids éviscéré), d'autre part, ont été calculées. Le nombre de branchiospines a été analysé en fonction de la taille de l'individu ce qui permet de différencier les deux espèces. En effet, ce caractère est l'élément clé de la détermination de l'espèce car en général, $A$. alosa possède plus de 90 branchiospines et $A$. fallax moins de 60 (SABATIE, BOISNEAU et ALEXANDRINO, 2000). Des valeurs moyennes ont été calculées par espèce. Les écailles récoltées ont été triées et nettoyées. Leur lecture a été effectuée en suivant le protocole de MENNESSON-BOISNEAU et BAGLINIERE (1992) concernant l'interprétation des différentes structures. Compte-tenu des observations de terrain concernant la reproduction des deux espèces, on peut raisonnablement fixer une date moyenne de ponte pour les deux espèces au $1^{\text {er }}$ juin. 57 écailles de grandes aloses et 34 d'aloses feintes ont pu être interprétées ce qui a permis de calculer un âge moyen de première remontée et d'établir la structure en âge.

\section{Caractéristiques génétiques}

Cette caractérisation a été effectuée par la méthode électrophorétique pour 48 grandes aloses et 30 aloses feintes.

\section{Récolte des données}

Des échantillons de foie, de sang et de muscle ont été prélevés sur tous les individus et conservés à $-80^{\circ} \mathrm{C}$. Ils ont ensuite été traités pour isoler les protéines qui ont été choisies en raison du caractère polymorphe des gènes qui les codent selon la méthode développée par ALEXANDRINO (1996). Les protéines séparées sont : 6 enzymes, une protéine plasmatique non identifiée et la chaîne $\alpha$ de l'hémoglobine.

Deux techniques de séparation ont été utilisées pour analyser les échantillons. La technique électrophorétique standard est actuellement la plus utilisée parce qu'elle permet d'analyser simultanément plusieurs dizaines d'individus et d'examiner le degré de variabilité intra-spécifique. La seconde technique de séparation est la focalisation électrique (IEF : isoelectric focusing) qui est une électrophorèse sur gel d'amidon contenant un gradient de $\mathrm{pH}$ (et non à $\mathrm{pH}$ constant) (ALEXANDRINO et BOISNEAU, 2000).

Les caractéristiques des protéines utilisées pour l'analyse génétique des échantillons des deux populations d'aloses de la Charente, le loci correspondant, la méthode de séparation utilisée, ainsi que le tissu dont les protéines ont été extraites, sont donnés dans le Tableau II.

\section{Traitement des données}

L'intérêt d'utiliser 8 marqueurs différents était de pouvoir traduire la variabilité individuelle génomique à l'aide des bandes révélées pour chaque extrait, les protéines étant la première expression des gènes (ALEXANDRINO et BOISNEAU, 2000). L'interprétation des gels d'électrophorèse consiste à relever, pour chaque loci, les phénotypes de chaque individu de la population étudiée qui sont traduits en terme de fréquence allélique au sein de la population. Les fréquences relatives théoriques des phénotypes ainsi que leurs fréquences absolues attendues sont ensuite calculées. Un test du $\chi^{2}$ permet de s'assurer que les populations suivent l'équilibre d'Hardy-Weinberg. 
Ces calculs sont effectués à l'aide du logiciel BIOSYS version 1.7 suivant les critères de Nei (1978). Les distributions des fréquences alléliques et les taux d'hétérozygotie des populations étudiées ont été calculés puis comparés à ceux obtenus antérieurement sur d'autres fleuves de la façade atlantique et de la Méditerranée. La différentiation génétique entre les populations a été analysée ensuite en terme de distance génétique de Nei (1978) par le même logiciel et mis sous forme de matrice et d'arbres phylogénétiques par le programme PHYLIP version 3.5.

\section{Tableau II}

Enzymes et protéines non enzymatiques des tissus des deux espèces d'aloses analysées par électrophorèse et IEF. Les locus auxquels ces protéines correspondent sont notés (d'après ALEXANDRINO, 1996 (1) et ALEXANDRINO et al., données non publiées (2)).

\section{Table II}

Enzymes and nonenzymatic proteins of fabrics of the two species of shads analyzed by electrophoresis and IEF. The loci to which these proteins correspond are noted (according to ALEXANDRINO, 1996 (1) and ALEXANDRINO et al., data not published (2)).

\begin{tabular}{lccc}
\hline Protéine & Loci & $\begin{array}{c}\text { Méthode de } \\
\text { séparation }\end{array}$ & $\begin{array}{c}\text { Matériel } \\
\text { biologique }\end{array}$ \\
\hline Adénosine Désaminase & ADA & IEF (1) & Muscle \\
Adénosine Déshydrogénase & ADH & Electrophorèse (1) & Foie \\
Phosphatase Acide & ACP-2 & IEF (2) & Foie \\
Enzyme Malique & ME-2 & Electrophorèse (2) & Foie \\
Mannose Phosphate Isomérase & MPI & Electrophorèse (2) & Foie \\
Alfa Amylase & AMY & Electrophorèse (2) & Plasma \\
Protéine Plasmatique non identifiée & PX & IEF (1) & Plasma \\
Chaîne $\alpha$ de l'hémoglobine & HBA & IEF (1) & Erythrocytes \\
\hline
\end{tabular}

\section{RÉSULTATS ET DISCUSSION}

\section{Front de migration et zones de reproduction}

En conditions hydrauliques normales, les aloses feintes et les grandes aloses sont bloquées relativement en aval du bassin, soit pour les aloses feintes au barrage de la Baine, en amont de Saintes et les grandes aloses en aval de Cognac à Crouin et parfois jusqu'à Chateauneuf. Mais lors de printemps pluvieux, comme en 1998, les barrages à clapet sont effacés et ainsi les aloses feintes sont remontées cette année là jusqu'à Crouin et les grandes aloses jusqu'à Ruffec en amont d'Angoulême (Figure 2). 
Des frayères actives de grandes aloses et d'aloses feintes ont été répertoriées en 1998 entre Saint Savinien et Angoulême (Figure 2). Cette année là, la plage temporelle de la reproduction des grandes aloses se situe entre la première quinzaine de mai et la première quinzaine de juin, celle des aloses feintes entre la seconde quinzaine de mai et la première quinzaine de juin.

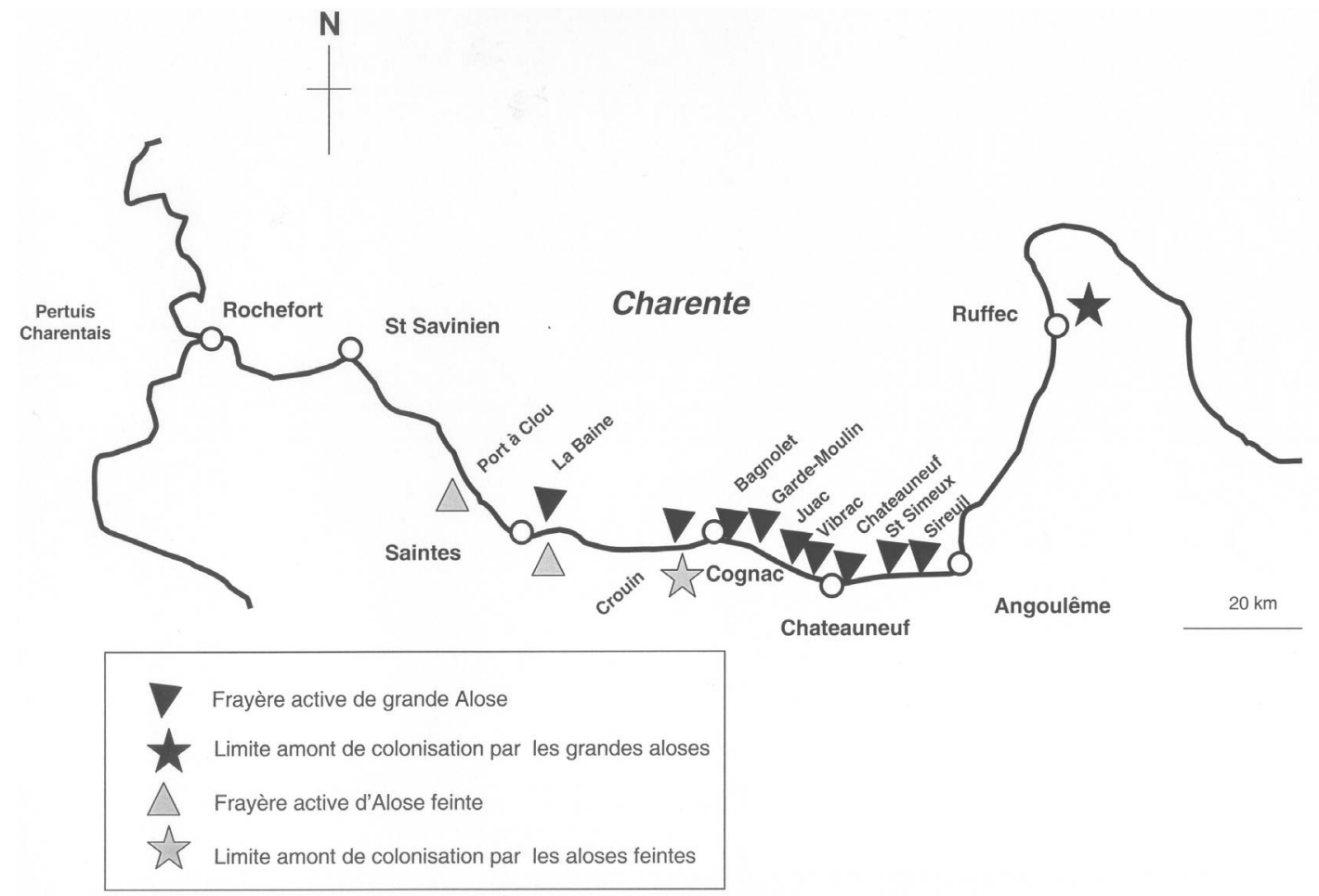

\section{Figure 2}

Répartition des frayères actives et potentielles de grande alose et d'alose feinte sur la Charente. Front de migration de ces deux espèces en 1998 (printemps pluvieux).

\section{Figure 2}

Distribution of the active and potential spawning grounds of allis shad and twaite shad on Charente river. Limits of the migration of the two species in 1998 (rainy spring).

\section{Caractéristiques biologiques}

\section{Nombre de branchiospines}

Le comptage du nombre de branchiospines du premier arc branchial de chacun des 88 individus échantillonnés permet de différencier les deux espèces au moyen d'une courbe de tri (Figure 3). L'étude de la régression linéaire entre les variables $\mathrm{Lt}$ et $\mathrm{Br}$ a montré qu'il n'apparaissait pas de corrélation significative (test $r$ ) entre ces deux variables, la valeur moyenne du nombre de branchiospines peut donc être retenue. 


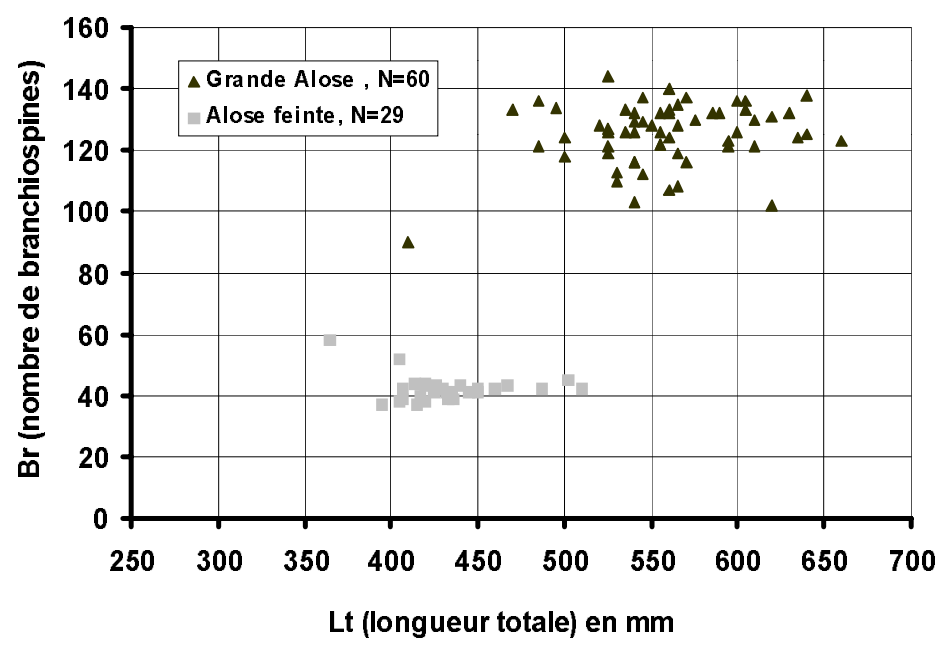

Figure 3

Relation entre la taille (Lt) et le nombre total de branchiospines chez les grandes aloses et aloses feintes de la Charente.

\section{Figure 3}

Relation between the size (Lt) and the total number of branchiospines in the allis shad and twaite shads of Charente.

\section{Morphologie}

La distribution des classes de taille (Lt) des aloses de la Charente échantillonnées montre que les femelles de grande alose et d'alose feinte ont des tailles moyennes supérieures à celles des mâles et sont les seules présentes pour les classes de taille les plus élevées (Figure 4). Pour les deux espèces, le dimorphisme est proche des $50 \mathrm{~mm}$ (Tableau III). Chez les grandes aloses le mode concerne les individus dont la taille est comprise entre 540 et $560 \mathrm{~mm}$ (23,33\% des grandes aloses échantillonnées). Pour les aloses feintes, c'est la classe $420-440 \mathrm{~mm}$ qui est la mieux représentée $(36,36 \%$ des aloses feintes échantillonnées). Les valeurs moyennes de la taille totale Lt ne présentent pas de différences significatives en fonction de l'espèce $(p>0,01)$.

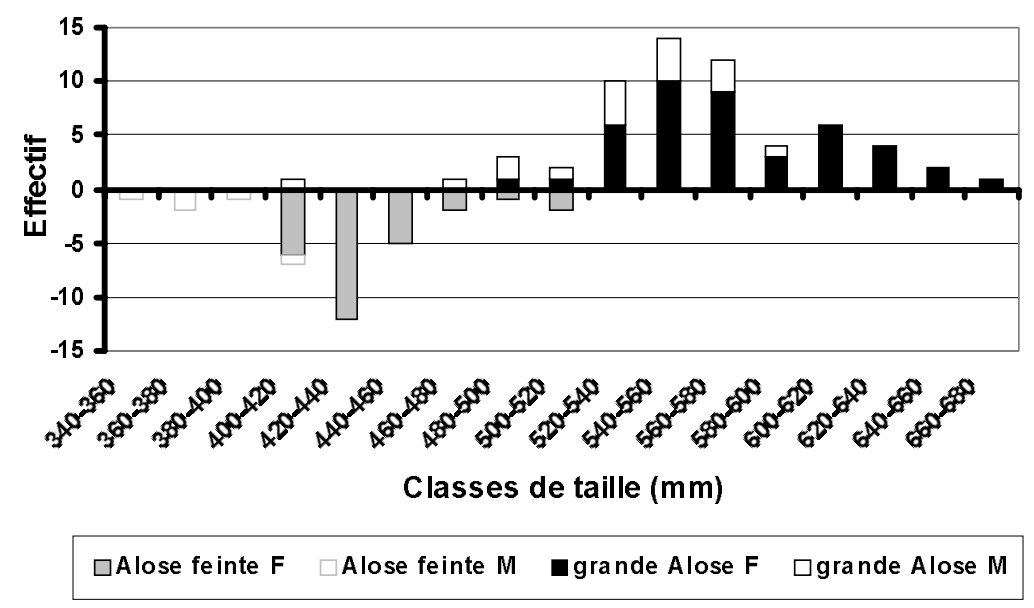

Figure 4

Structures en taille (Lt) des grandes aloses et aloses feintes de la Charente.

\section{Figure 4}

Length distribution (Lt) of allis shad and twaite shad of Charente. 


\section{Tableau III}

Longueur totale moyenne (Lt moy en $\mathrm{mm}$ ), écart-type (E-t), intervalle de confiance (alpha $=0.05$ ), effectif $(\mathrm{N})$, minimum (Lt $\mathrm{min}$ ) et maximum (Lt $\max$ ) observés pour chaque échantillon par sexe et état sexuel pour les 2 espèces (Aa : grande Alose, Af : Alose feinte) sur le bassin de la Charente.

Table III

Average total length (Lt moy in $\mathrm{mm}$ ), standard deviation (E-t), confidence interval (alpha = 0.05), effective ( $\mathrm{n}$ ), minimum (Lt min) and maximum (Lt max) observed for each sample by sex and sexual state for the 2 species (Aa: Allis shad, Af: twaite shad) on the basin of Charente.

\begin{tabular}{ccccccccc}
\hline Bassin & Espèce & Sexe & Lt Moy & E-t & Int-conf & $\mathbf{N}$ & Lt min & Lt max \\
\hline Charente & Aa & M & $\mathbf{5 2 7 . 1}$ & 44.5 & 21.2 & 17 & 410 & 595 \\
& Aa & F & $\mathbf{5 7 0 . 2}$ & 40.8 & 12.2 & 43 & 485 & 660 \\
\hline \multirow{2}{*}{ Charente } & Af & M & $\mathbf{3 7 6 . 0}$ & 29.2 & 25.6 & 5 & 340 & 415 \\
& Af & F & $\mathbf{4 3 8 . 2}$ & 27.3 & 12.1 & 28 & 405 & 510 \\
\hline
\end{tabular}

Age

Chez les grandes aloses, les femelles comme les mâles apparaissent dès l'âge de trois ans (Figure 5), les plus vieux atteignent 7 ans. La majorité des mâles et des femelles ont 5 ans. Les aloses feintes femelles apparaissent aussi à 3 ans, alors que les mâles ont 4 ans. La majorité des femelles d'aloses feintes a 4 ans.

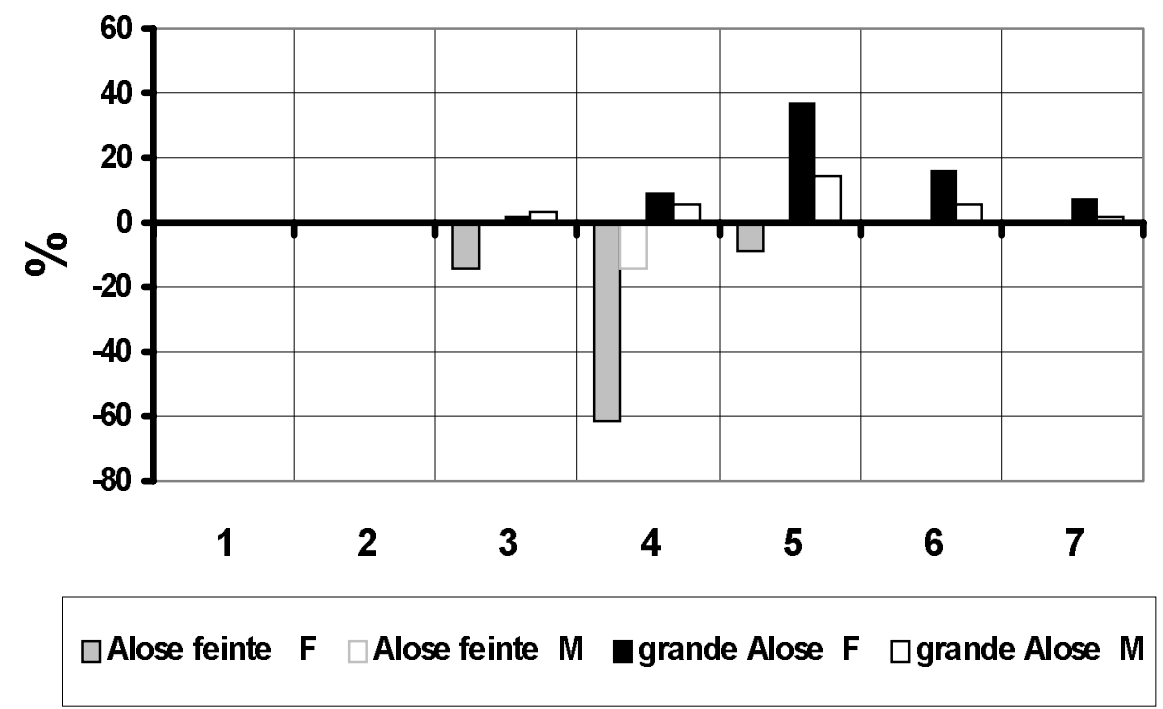

Figure 5

Structure en âge des grandes aloses et aloses feintes de la Charente.

Figure 5

Distribution of the ages of the allis shad and twaite shad of Charente. 
La croissance brute en longueur est calculée (Figure 6), les femelles de grande alose et d'alose feinte aux mêmes âges ont des tailles supérieures à celles des mâles. $\mathrm{A}$ âge égal, les grandes aloses ont des tailles supérieures à celles des aloses feintes. Les taux de croissance des grandes aloses et des aloses feintes femelles sont sensiblement les mêmes. $47 \%$ des aloses feintes échantillonnées présentent plusieurs marques de reproduction : $12,5 \%$ des femelles se sont déjà reproduites une fois à l'âge moyen de 3 ans et 3,5\% se sont reproduites deux fois aux âges 3 et 4 ans. Chez les mâles, $40 \%$ se sont déjà reproduits une fois à l'âge moyen de 3 ans. Chez les grandes aloses, aucune marque de reproduction antérieure n'a été détectée.

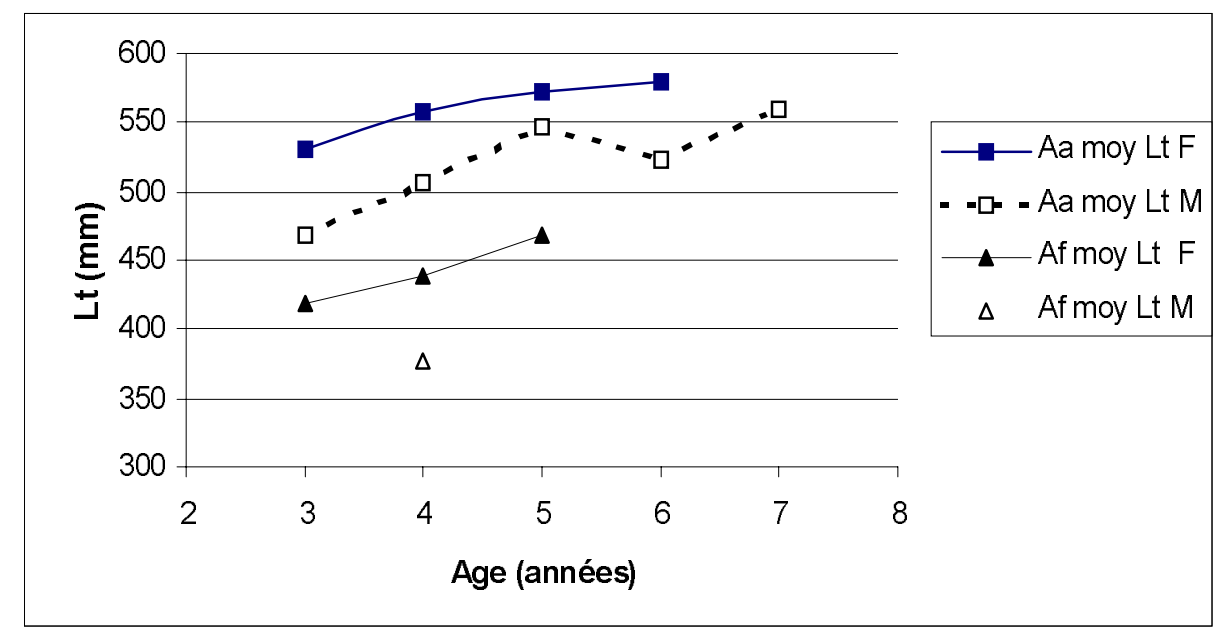

\section{Figure 6}

Relation longueur moyenne / âge des populations de grande Alose (Aa) et d'Alose feinte (Af) de la Charente.

\section{Figure 6}

Relation average length and age of the populations of allis shad ( $\mathrm{Aa})$ and twaite shad (Af) of Charente.

\section{Caractéristiques génétiques}

Les résultats des analyses génétiques effectuées sur les échantillons de la Charente révèlent les allèles rencontrés pour les différents loci habituellement présents chez les deux espèces d'aloses des populations atlantiques. Dans les deux populations et pour tous les locus, sauf pour l'ADA des aloses feintes, l'équilibre de Hardy-Weinberg est bien respecté $(p>0.05)$.

Pour les différents locus (Tableaux IV et $V$ ) nous pouvons observer: un allèle diallélique pour lequel l'un des deux allèles est fixé, le taux d'hétérozygotie de ce loci est alors nul. Des locus dialléliques ou polyalléliques pour lesquels un des allèles est largement majoritaire et les autres allèles sont rares, le taux d'hétérozygotie est alors très faible. Des locus dialléliques pour lesquels les deux allèles sont en proportion presque égale. Un loci multiallélique (ADA) proche de l'équilibre et dont le taux d'hétérozygotie est alors maximal. 


\section{Tableau IV}

Grande Alose de la Charente. Taille de l'échantillon (N), nombre d'individus observés ( $\mathrm{N}$ obs) et théoriques ( $\mathrm{N}$ att) pour chaque phénotype, valeur du $\chi^{2}$, du degré de liberté (ddl) et probabilité (p) du test de conformité du test de HardyWeinberg à chacun des 8 locus étudiés, fréquence allélique (freq. allélique) pour chacun des allèles rencontrés (Echantillon global = 55 grandes aloses).

Table IV

Allis shad of Charente. Size of the sample (N), a number of individuals observed ( $\mathrm{N}$ obs) and theoretical ( $\mathrm{N}$ att) for each phenotypes, value of the $\chi^{2}$, degree of freedom (ddl) and probability (p) of the test of conformity of the test of HardyWeinberg to each of the 8 loci studied, allelic frequency (freq. allelic) for each allele (total Sample $=\mathbf{5 5}$ allis shad).

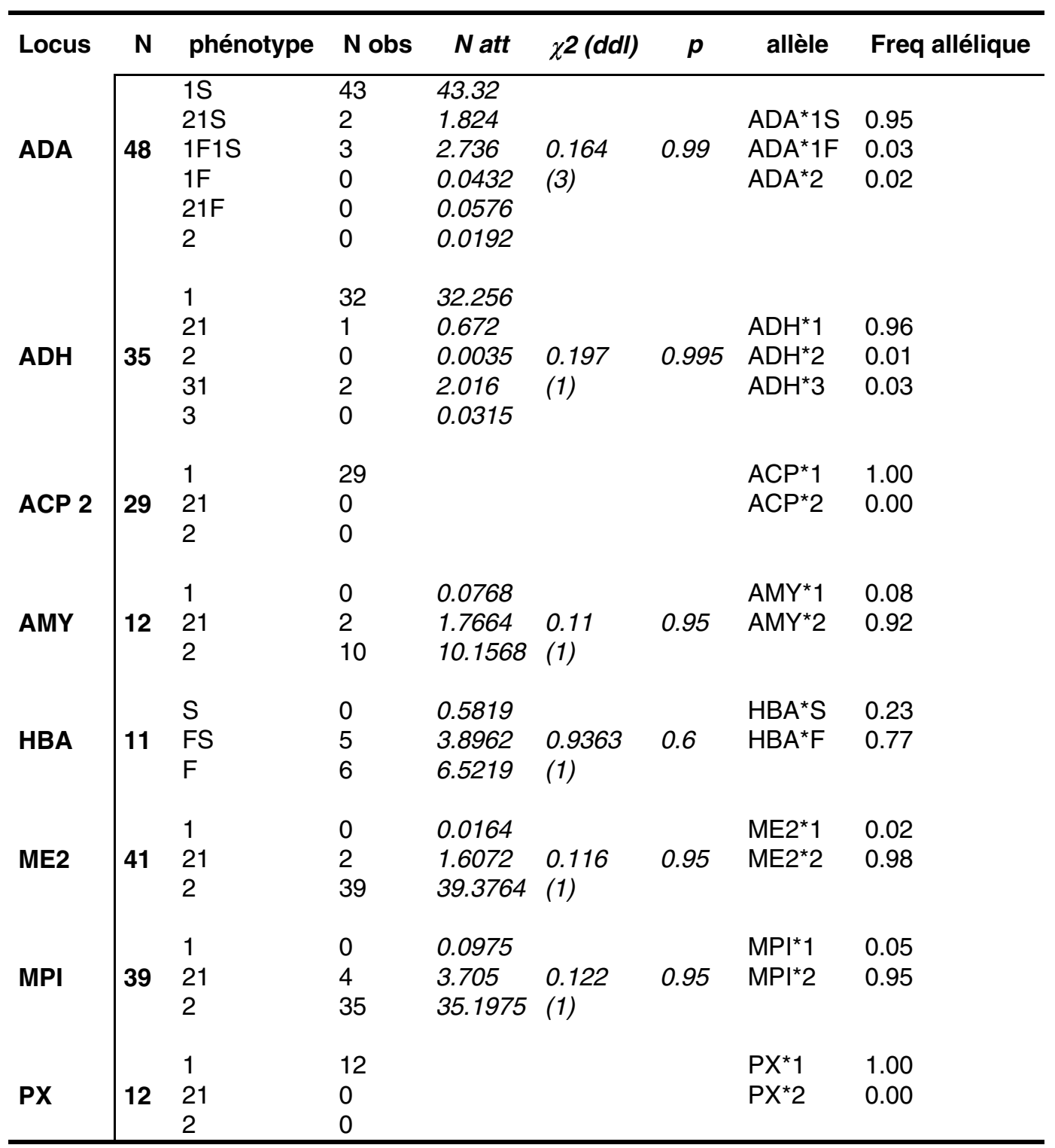




\section{Tableau V}

Alose feinte de la Charente. Taille de l'échantillon (N), nombre d'individus observés ( $\mathrm{N}$ obs) et théoriques ( $\mathrm{N}$ att) pour chaque phénotype, valeur du $\chi^{2}$, du degré de liberté (ddl) et probabilité (p) du test de conformité du test de HardyWeinberg à chacun des 8 locus étudiés, fréquence allélique (freq. allélique) pour chacun des allèles rencontrés (Echantillon global $=35$ aloses feintes).

\section{Table V}

Twaite shad of Charente. Size of the sample $(\mathrm{N})$, a number of individuals observed ( $\mathrm{N}$ obs) and theoretical $\left(\mathrm{N}\right.$ att) for each phenotypes, value of the $\chi^{2}$, degree of freedom (ddl) and probability (p) of the test of conformity of the test of Hardy-Weinberg to each of the 8 loci studied, allelic frequency (freq. allelic) for each allele (total sample $=35$ twaite shads).

\begin{tabular}{|c|c|c|c|c|c|c|c|c|}
\hline Locus & $\mathbf{N}$ & phénotype & $\mathrm{N}$ obs & $N$ att & $\chi^{2}(d d l)$ & $p$ & allèle & Freq allélique \\
\hline \multirow{6}{*}{ ADA } & \multirow{6}{*}{23} & $1 \mathrm{~S}$ & 0 & 2.8175 & & \multirow{6}{*}{$\begin{array}{l}0.000 \\
1\end{array}$} & & \\
\hline & & $21 S$ & 16 & 8.05 & & & $\mathrm{ADA}^{*} 1 \mathrm{~S}$ & 0.35 \\
\hline & & $1 \mathrm{~F} 1 \mathrm{~S}$ & 0 & 2.415 & 23.004 & & $\mathrm{ADA}^{*} 1 \mathrm{~F}$ & 0.15 \\
\hline & & $1 \mathrm{~F}$ & 0 & 0.5175 & (3) & & $\mathrm{ADA}^{*} 2$ & 0.50 \\
\hline & & $21 \mathrm{~F}$ & 7 & 3.45 & & & & \\
\hline & & 2 & 0 & 5.75 & & & & \\
\hline \multirow{5}{*}{ ADH } & \multirow{5}{*}{21} & 1 & 11 & 10.5861 & & & & \\
\hline & & 21 & 8 & 8.6478 & & & $\mathrm{ADH}^{*} 1$ & 0.71 \\
\hline & & 2 & 2 & 1.7661 & 0.095 & 0.8 & $\mathrm{ADH}^{\star 2}$ & 0.29 \\
\hline & & 31 & 0 & 0 & (1) & & $\mathrm{ADH}^{*} 3$ & 0.00 \\
\hline & & 3 & 0 & 0 & & & & \\
\hline \multirow{3}{*}{ ACP 2} & \multirow{3}{*}{24} & 1 & 23 & 23.0496 & & & $\mathrm{ACP}^{*} 1$ & 0.98 \\
\hline & & 21 & 1 & 0.9408 & 0.013 & 0.95 & $A C P * 2$ & 0.02 \\
\hline & & 2 & 0 & 0.0096 & (1) & & & \\
\hline \multirow{3}{*}{ AMY } & \multirow{3}{*}{30} & 1 & 0 & & & & $\mathrm{AMY}^{* 1}$ & 0.00 \\
\hline & & 21 & 0 & & & & $\mathrm{AMY}^{\star 2} 2$ & 1.00 \\
\hline & & 2 & 30 & & & & & \\
\hline \multirow{3}{*}{ HBA } & \multirow{3}{*}{25} & $S$ & 25 & & & & $\mathrm{HBA}^{*} \mathrm{~S}$ & 1.00 \\
\hline & & FS & 0 & & & & $\mathrm{HBA}^{*} \mathrm{~F}$ & 0.00 \\
\hline & & $\mathrm{F}$ & 0 & & & & & \\
\hline \multirow{3}{*}{ ME2 } & \multirow{3}{*}{17} & 1 & 0 & 0.0153 & & & ME2*1 & 0.03 \\
\hline & & 21 & 1 & 0.9894 & 0.015 & 0.95 & ME2*2 & 0.97 \\
\hline & & 2 & 16 & 15.9953 & (1) & & & \\
\hline \multirow{3}{*}{ MPI } & \multirow{3}{*}{19} & 1 & 18 & 17.877 & & & $\mathrm{MPI}^{*} 1$ & 0.97 \\
\hline & & 21 & 1 & 1.1058 & 0.028 & 0.95 & $\mathrm{MPI}^{\star 2}$ & 0.03 \\
\hline & & 2 & 0 & 0.0171 & (1) & & & \\
\hline \multirow{3}{*}{ PX } & \multirow{3}{*}{25} & 1 & 25 & & & & $P X^{*} 1$ & 1.00 \\
\hline & & 21 & 0 & & & & $P X^{\star} 2$ & 0.00 \\
\hline & & 2 & 0 & & & & & \\
\hline
\end{tabular}


Les locus MPI, ADA et HBA sont les plus appropriés pour discriminer les deux espèces (ALEXANDRINO, 1996). En effet, l'allèle MPI*1 est rare chez les grandes aloses et fréquent chez les aloses feintes. On retrouve des caractéristiques similaires pour les allèles $\mathrm{HBA}^{\star} \mathrm{S}$ et $\mathrm{ADA}^{\star} 2$. Les loci $\mathrm{PX}, \mathrm{ME} 2, \mathrm{AMY}$ et $\mathrm{ACP} 2$ ne révèlent pas de différences significatives en terme de fréquences alléliques entre les deux populations étudiées.

Parmi les 8 locus présentés, 5 ont déjà été étudiés (ADA, ADH, HBA, MPI et PX) chez plusieurs populations de grandes aloses et d'aloses feintes (ALEXANDRINO, 1996 ; LE CORRE, 1999). Les résultats obtenus en terme de fréquence allélique et de taux d'hétérozygotie peuvent donc être comparés (Tableau VI).

\section{Tableau VI}

Fréquences alléliques moyennes rencontrées chez les grandes aloses et les aloses feintes de l'Atlantique (ALEXANDRINO, 1996), du Rhône (LE CORRE, 1998) et chez les deux populations d'aloses nouvellement étudiées. Hétérozygotie moyenne observée (Hs) pour chaque population et pour les populations de l'Atlantique Est (1) et (2).

Table VI

Average allelic frequencies of the allis shad and the twaite shads of the EastAtlantic rivers (ALEXANDRINO, 1996), of the Rhone river (LE CORRE, 1998) and of the two populations of studied shads. Average real heterozygoty (Hs) for each population and the populations of the East-Atlantic (1) et (2).

\begin{tabular}{|c|c|c|c|c|c|c|}
\hline & & $\begin{array}{c}\text { A. alosa } \\
\text { moyenne } \\
5 \text { populations } \\
\text { Atlantiques (1) }\end{array}$ & $\begin{array}{l}\text { A. alosa } \\
\text { Charente }\end{array}$ & $\begin{array}{c}\text { A. fallax } \\
\text { moyenne } \\
6 \text { populations } \\
\text { Atlantiques (2) }\end{array}$ & $\begin{array}{l}\text { A. fallax } \\
\text { Charente }\end{array}$ & $\begin{array}{c}\text { A. fallax } \\
\text { rhodanensis } \\
\text { Méditerranée }\end{array}$ \\
\hline & *1S & 0.98 & 0.95 & 0.08 & 0.35 & 0.01 \\
\hline \multirow[t]{3}{*}{ ADA } & $* 1 F$ & 0.01 & 0.03 & 0.50 & 0.15 & 0.96 \\
\hline & *2 & 0.01 & 0.02 & 0.42 & 0.50 & 0.00 \\
\hline & *3 & 0.00 & & 0.00 & & 0.03 \\
\hline \multirow[t]{3}{*}{ ADH } & *1 & 1.00 & 0.96 & 0.54 & 0.71 & 1.00 \\
\hline & *2 & 0.00 & 0.01 & 0.46 & 0.29 & 0.00 \\
\hline & *3 & 0.00 & 0.03 & 0.00 & 0.00 & \\
\hline \multirow[t]{2}{*}{ HBA } & ${ }^{*} S$ & 0.06 & 0.23 & 0.94 & 1.00 & 0.99 \\
\hline & ${ }^{\star} \mathrm{F}$ & 0.94 & 0.77 & 0.06 & 0.00 & 0.01 \\
\hline \multirow[t]{2}{*}{ MPI } & *1 & 0.03 & 0.05 & 0.78 & 0.97 & 1.00 \\
\hline & *2 & 0.97 & 0.95 & 0.22 & 0.03 & 0.00 \\
\hline \multirow[t]{2}{*}{ PX } & *1 & 0.99 & 1.00 & 0.70 & 1.00 & 0.55 \\
\hline & *2 & 0.01 & 0.00 & 0.30 & 0.00 & 0.45 \\
\hline \multicolumn{2}{|c|}{ Hs } & 0.04 & 0.10 & 0.38 & 0.15 & 0.12 \\
\hline
\end{tabular}

(1) populations de grande alose : Castelo Bode, Lima, Mondego, Garonne et Ecosse.

(2) populations d'alose feinte : Lima, Mondego, Tage, Mira, Guadiana et Ecosse.

(1) Allis shad populations : Castelo Bode, Lima, Mondego, Garonne and Scotland.

(2) Twaite shad populations : Lima, Mondego, Tage, Mira, Guadiana and Scotland. 
La population de grande alose de la Charente présente des distribution de fréquences alléliques relativement proches de celles correspondant à la moyenne des distributions des autres populations de l'Atlantique. La population de grande alose de la Charente se distinguant cependant par une distribution plus variable du locus HBA ce qui se traduit par un taux d'hétérozygotie le plus élevé pour ce locus $(0,35)$, le locus HBA* $F$ étant presque fixé pour l'ensemble des populations atlantiques (Tableau VI). L'hétérozygotie moyenne de la population de grande alose de la Charente est supérieure à celle de la moyenne des populations atlantiques. Le cas du loci ADH est intéressant, car un allèle $\left(\mathrm{ADH}^{\star} 3\right)$ a été détecté dans la population de grandes aloses de la Charente et il est présent avec une fréquence supérieure à celle de l'allèle $\mathrm{ADH}^{\star} 2$.

Globalement, les aloses feintes de la Charente apparaissent beaucoup moins polymorphes que celles de l'ensemble des populations atlantiques étudiées. Le taux d'hétérozygotie moyen de la population de Charente atteint en effet 0,15 alors que celui de la moyenne des populations atlantiques est de 0,39. Ce qui est normal car en prenant en compte toutes les populations d'aloses feintes atlantiques leurs polymorphismes s'ajoutent ce qui augmente le taux d'hétérozygotie moyen. En revanche, les hétérozygoties moyennes des populations d'aloses feintes de la Charente et du Rhône sont comparables (Hs Rhône $=0,12$; Hs Charente $=0,15$ ). Cela s'explique par deux niveaux de différence des distributions des fréquences alléliques. Tout d'abord, sur les 5 locus étudiés chez l'ensemble des populations atlantiques, aucun ne présente d'allèle fixé, alors que les aloses feintes de la Charente, sur 8 locus étudiés, présentent 3 allèles fixés: AMY*2, $\mathrm{HBA}^{*} S$ et $P X^{\star} 1$ et 3 allèles très fortement majoritaires (fréq alléliques $\geq 0,97$ ) qui sont : ACP2*1, ME2*2 et MPI*1. Loci par loci, les différences de distribution les plus importantes concernent les locus ADA et PX.

Les distances génétiques entre les deux populations de la Charente sont incluses dans une matrice de Nei construite pour les 5 loci choisis et contenant par ailleurs les populations de la façade Atlantique (ALEXANDRINO, 1996), de l'Aulne (VERON, 1999), de la Méditerranée (LE CORRE, 1999) et du Maroc (SABATIE, données non publiées), (Tableau VII). Un dendrogramme permet de présenter les liens entre ces populations (Figure 7). Les 16 populations d'aloses représentées peuvent être organisées en 3 groupes distincts :

- Les aloses feintes de la Méditerranée et de la côte atlantique du Maroc, comprenant les 4 populations d'aloses feintes du Rhône, de l'Aude, de l'Hérault et du Sebou. Les aloses feintes du Rhône et du Sebou sont très proches ( $\mathrm{D}$ Nei $=0,009$ ), celles de l'Aude et de l'Hérault seraient confondues (D Nei $=0$ ).

- Les aloses feintes de la Charente et du Portugal, comprenant 5 populations correspondant aux aloses feintes de la Charente, du Mondego, du Lima, du Tage et du Guadiana. La population de Charente étant proche de celle du Mondego ( $\mathrm{D} \mathrm{Nei}=0,020$ ).

- Les grandes aloses du Portugal et de la France, comprenant 6 populations correspondant aux fleuves portugais (Zezere (C Bode), Mondego, Lima) et français (Garonne, Charente et Aulne). Les distances génétiques entre ces populations sont très faibles ( $\mathrm{D} \mathrm{Nei}<0,008)$, ce qui confirme le caractère très peu polymorphe des populations de grandes aloses. Les populations de la Garonne, de la Charente et celles de l'Aulne sont très proches $(\mathrm{D}$ Nei $=0,001)$ comme le sont aussi les grandes aloses portugaises.

- Une population d'hybrides du fleuve Lima proche du groupe des grandes aloses. 


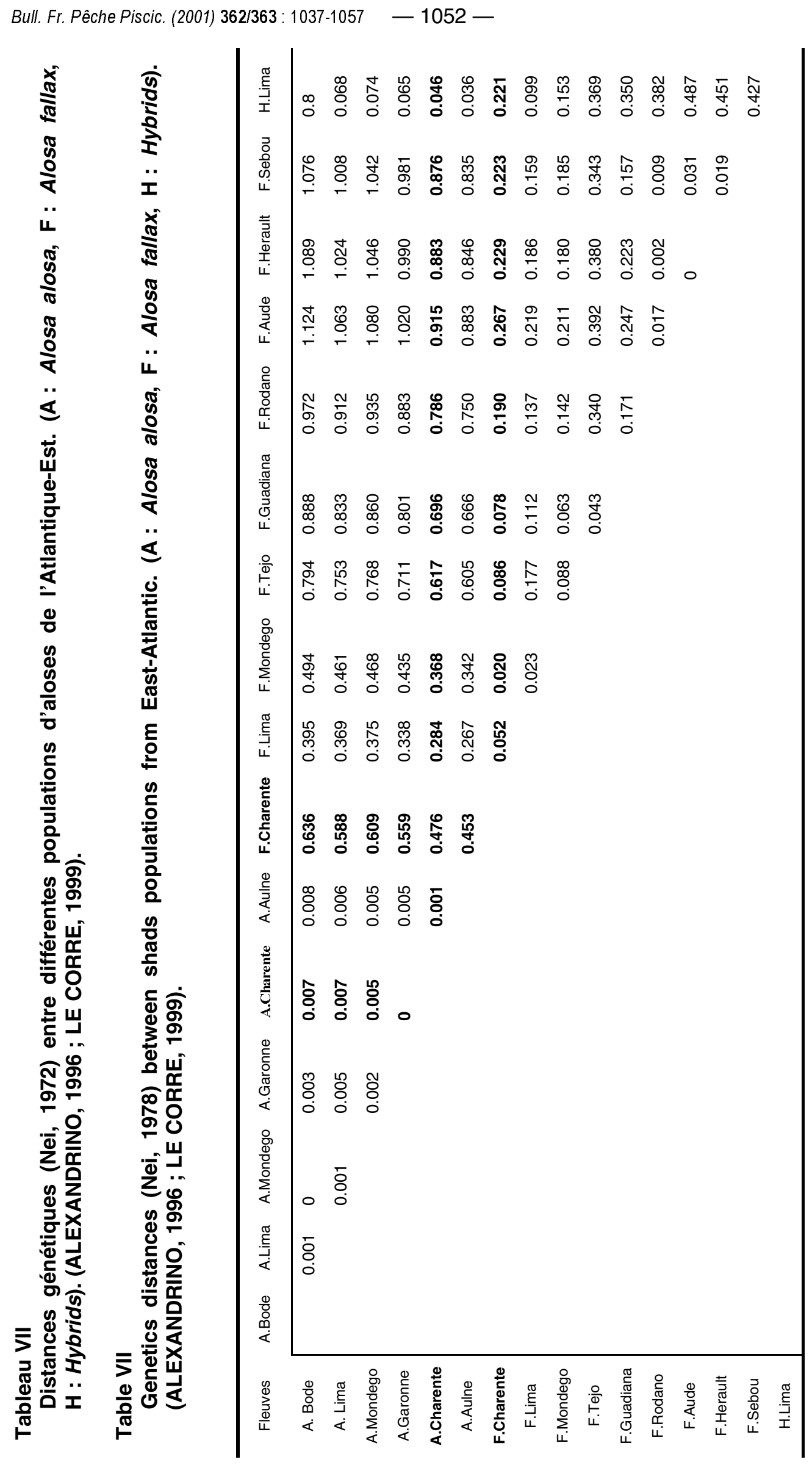



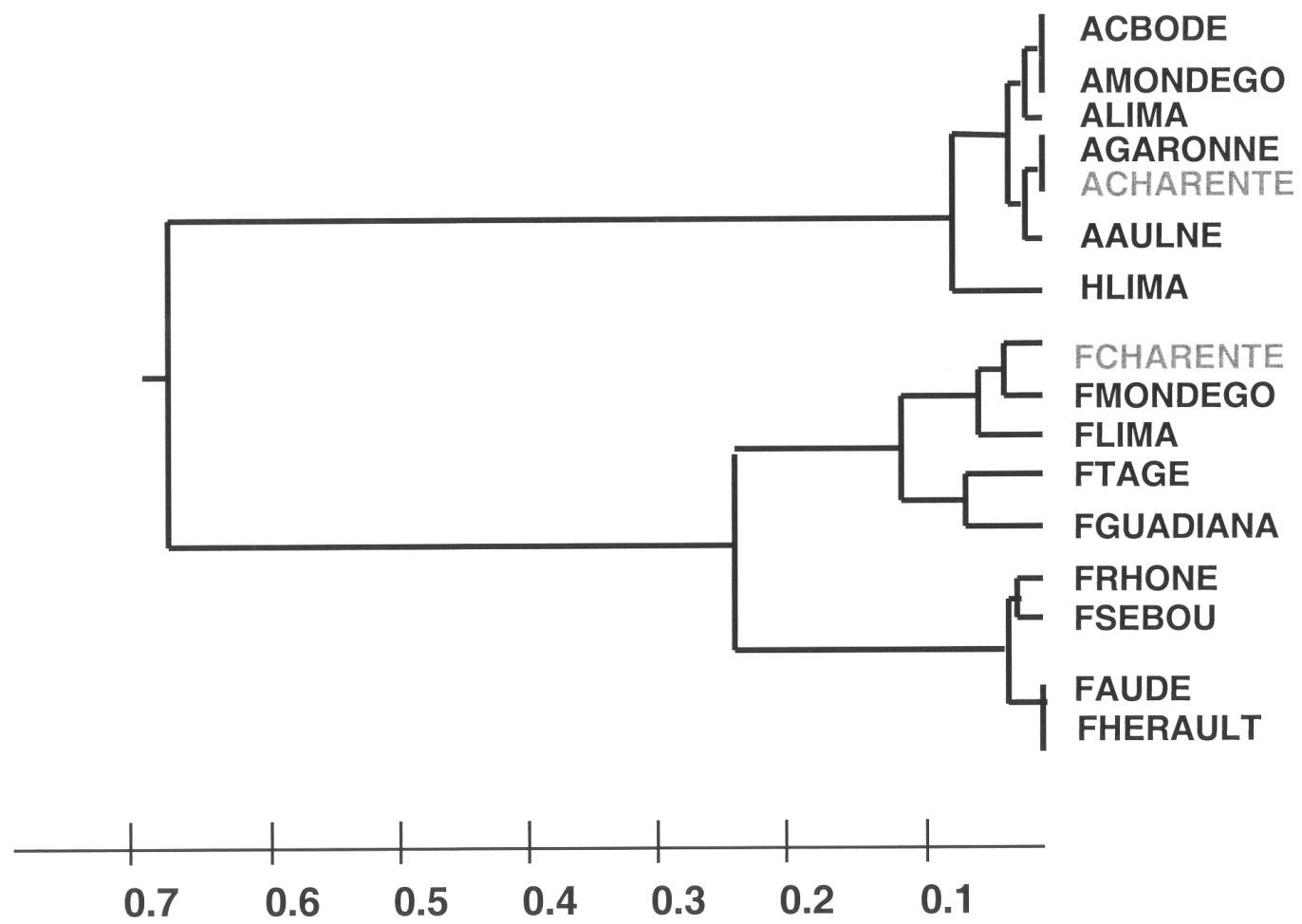

\section{Figure 7}

Arbre phylogénétique obtenu selon la méthode UPGMA à partir de la matrice des distances génétiques de Nei. (5 loci choisis : ADA, ADH, HBA, MPI et PX) A : grande Alose ; F : Alose feinte et H : Hybride.

\section{Figure 7}

Phylogenetic tree obtained according to method UPGMA from the matrix of the genetic distances from Nei. (5 loci selected : ADA, ADH, HBA, MPI and PX) A : Allis shad ; F : Twaite shad and $H$ : Hybrid.

\section{CONCLUSION}

Les deux années étudiées, 1997 et 1998, sont caractérisées par des conditions hydrauliques printanières extrêmes et ont permis d'établir les situations limites des fronts de migration et d'observer un nombre plus élevé de frayères de grande aloses réparties sur l'ensemble du fleuve colonisé. Le nombre de frayères actives ainsi que celui de bulls par frayère pourraient être traduits en un indice d'abondance des géniteurs compte-tenu de l'absence de tout dispositif de contrôle des migrations. Le stock d'aloses de la Charente est exploité par pêche à la ligne et pêche professionnelle en estuaire. Une étude des poissons migrateurs de la Charente et de la Seudre (historique des captures, état des stocks par enquête halieutique, potentialités et actions de réhabilitation) est actuellement en projet et devrait fournir des arguments supplémentaires sur la nécessité d'équiper les nombreux ouvrages de la Charente en passes à poissons afin de rendre transparent cet axe migratoire et ainsi permettre une progression rapide des géniteurs jusqu'aux zones de reproduction.

La population de grandes aloses de la Charente présente une valeur moyenne du nombre de branchiospines (125) proche de celui des autres populations de la façade atlantique. Le nombre de branchiospines est sensiblement le même chez toutes les 
populations d'aloses feintes $(38<\mathrm{Br}<46)$ (Tableau VIII). Deux populations se distinguent cependant des autres : celle de la Loire $(\mathrm{Br}=48,5)$ et celle du fleuve $\mathrm{Lima}(\mathrm{Br}=49,9)$ avec des valeurs moyennes du nombre de branchiospines élevées. Les aloses feintes de la Charente présentent un nombre moyen de branchiospines (42) relativement proche de celui de la Garonne $(42,4)$, ce rapprochement devra être confirmé par un échantillonnage plus important.

\section{Tableau VIII}

Valeurs moyennes de la variable méristique: nombre de branchiospines du premier arc branchial gauche des populations de grande alose (Aa) et d'alose feinte (Af) de l'Atlantique Est et de la Méditerranée ouest.

\section{Table VIII}

Average values of the meristic variable: numbers branchiospines of the first left branchial arch of the populations of allis shad (Aa) and twaite shads (Af) of the East Atlantic and the Western Mediterranean.

\begin{tabular}{|c|c|c|c|c|c|c|}
\hline Bassin & Espèce & auteurs & année & Br moy & $\mathbf{N}$ & écart-type \\
\hline Orne & $\mathrm{Aa}$ & VERON & 1999 & 112 & 6 & 13 \\
\hline Vire & $\mathrm{Aa}$ & VERON & 1999 & 123 & 4 & 8 \\
\hline Aulne & $\mathrm{Aa}$ & VERON & 1999 & 112 & 18 & 11 \\
\hline Blavet & Aa & VERON & 1999 & 121 & 3 & 4 \\
\hline Vilaine & $\mathrm{Aa}$ & VERON & 1999 & 119 & 9 & 16 \\
\hline Loire & $\mathrm{Aa}$ & $\begin{array}{c}\text { MENNESSON-BOISNEAU } \\
\text { et BOISNEAU }\end{array}$ & 1990 & 122.1 & 681 & 10.27 \\
\hline Loire & $\mathrm{Aa}$ & DOUCHEMENT & 1981 & 129.3 & 131 & 6.66 \\
\hline Charente & $\mathrm{Aa}$ & Présent travail & & 125 & 57 & 10 \\
\hline Garonne & $\mathrm{Aa}$ & DOUCHEMENT & 1981 & 127.5 & 107 & 5.64 \\
\hline Adour & $\mathrm{Aa}$ & DOUCHEMENT & 1981 & 123.8 & 138 & 4.76 \\
\hline Lima (Portugal) & $\mathrm{Aa}$ & ALEXANDRINO & 1996 & 133.4 & 106 & 9.77 \\
\hline Douro (Port.) & $\mathrm{Aa}$ & ALEXANDRINO & 1996 & 123.8 & 204 & 10.73 \\
\hline Mondego (Port.) & $\mathrm{Aa}$ & ALEXANDRINO & 1996 & 124.3 & 20 & 12.40 \\
\hline Castelo do Bode (Port.) & $\mathrm{Aa}$ & ALEXANDRINO & 1996 & 142.0 & 36 & 5.50 \\
\hline Sebou (Maroc) & $\mathrm{Aa}$ & SABATIE & 1993 & 109.9 & 303 & 6.84 \\
\hline Rhône & & DOUCHEMENT & 1981 & 69.8 & 20 & 12.10 \\
\hline Aude & & DOUCHEMENT & 1981 & 72.3 & 12 & 4.12 \\
\hline Loire & Af & $\begin{array}{c}\text { MENNESSON-BOISNEAU } \\
\text { et BOISNEAU }\end{array}$ & 1990 & 48.5 & 53 & 4.77 \\
\hline Loire & Af & DOUCHEMENT & 1981 & 44.9 & 140 & 1.70 \\
\hline Charente & Af & Présent travail & & 42 & 28 & 4 \\
\hline Garonne & Af & DOUCHEMENT & 1981 & 42.4 & 231 & 1.58 \\
\hline Adour & Af & DOUCHEMENT & 1981 & 47.5 & 39 & 2.25 \\
\hline Lima (Portugal) & Af & ALEXANDRINO & 1996 & 49.9 & 62 & 4.64 \\
\hline Douro (Port.) & Af & ALEXANDRINO & 1996 & 42.2 & 89 & 2.00 \\
\hline Mondego (Port.) & Af & ALEXANDRINO & 1996 & 46.4 & 32 & 2.59 \\
\hline Tage (Port.) & Af & ALEXANDRINO & 1996 & 41.3 & 86 & 2.18 \\
\hline Mira (Port.) & Af & ALEXANDRINO & 1996 & 40.2 & 8 & 1.04 \\
\hline Guadianna (Port.) & Af & ALEXANDRINO & 1996 & 41.9 & 43 & 1.32 \\
\hline Sebou (Maroc) & Af & SABATIE & 1993 & 41.7 & 318 & \\
\hline Loukos (Maroc) & Af & SABATIE & 1993 & 41.9 & 59 & \\
\hline El Kansara (Lac maroc) & Af & SABATIE & 1993 & 38.4 & 18 & \\
\hline Rhône & Af & BAGLINIERE et al. & 1996 & 40 & 32 & 2.35 \\
\hline Rhône & Af & LE CORRE et al. & 1996 & 38.2 & 316 & 2.00 \\
\hline Aude & Af & DOUCHEMENT & 1981 & 42.3 & 388 & 2.93 \\
\hline
\end{tabular}

La population de grande Alose de la Charente (mâles et femelles) a une taille moyenne supérieure à celle des populations situées sur les bassins plus septentrionaux (Aulne, Vilaine et Loire) et proche de celle de la Gironde pour les deux sexes (Tableau IX). Les mâles ont toujours une taille moyenne inférieure à celle des femelles (sauf dans le cas 
du lac de Castelo Bode ). II semblerait que les écarts de croissance potentielle entre les populations situées au nord et au sud de l'aire de répartition de la grande Alose soient liés à la différence de localisation et de caractéristiques physiques des zones d'engraissement. En effet, les aloses marocaines sont présentes toutes l'année dans une zone d'upwelling centrée entre Essaouira et Agadir (SABATIÉ, 1993). Il en est de même pour les grandes aloses portugaises qui semblent bénéficier d'upwellings estivaux au large des côtes de la façade atlantique de ce pays (ALEXANDRINO, 1996).

\section{Tableau IX}

Taille moyenne (Lt moy en $\mathrm{mm}$ ), écart-type (E-t), effectif ( $\mathrm{N}$ ), minimum (Lt min) et maximum (Lt max) observés pour chaque échantillon par bassin, espèce (Aa : grande Alose, Af : Alose feinte) et sexe.

\section{Table IX}

length average (Lt moy in $\mathrm{mm}$ ), standard deviation (E-t), effective (n), minimum (Lt min) and maximum (Lt max) observed for each sample by river, species (Aa : Allis shad, Af : twaite shad) and sex.

\begin{tabular}{|c|c|c|c|c|c|c|c|c|}
\hline Bassin & Auteurs & Espèce & Sexe & Lt Moy & E-t & $\mathbf{N}$ & Lt min & Lt max \\
\hline \multirow{2}{*}{ Aulne } & VERON, 1999 & $\mathrm{Aa}$ & $\mathrm{F}$ & 552.5 & 12.5 & 8 & 540 & 575 \\
\hline & & $\mathrm{Aa}$ & M & 459.3 & 29.5 & 14 & 420 & 515 \\
\hline \multirow[t]{2}{*}{ Vilaine } & VERON, 1999 & $\mathrm{Aa}$ & $\mathrm{F}$ & 549.3 & 36.9 & 7 & 520 & 605 \\
\hline & & $\mathrm{Aa}$ & M & 473.0 & 39.5 & 4 & 425 & 517 \\
\hline \multirow[t]{2}{*}{ Loire } & MENNESSON-BOISNEAU, 1990 & $\mathrm{Aa}$ & M & 484 & 39 & 1172 & 339 & 635 \\
\hline & & $\mathrm{Aa}$ & $\mathrm{F}$ & 556 & 39 & 1041 & 414 & 680 \\
\hline \multirow[t]{2}{*}{ Charente } & & $\mathrm{Aa}$ & $\mathbf{F}$ & 570.2 & 40.8 & 43 & 485 & 660 \\
\hline & & $\mathbf{A a}$ & M & 527.1 & 44.5 & 17 & 410 & 595 \\
\hline \multirow[t]{2}{*}{ Gironde } & TAVERNY, 1991 & $\mathrm{Aa}$ & M & 533 & 29 & 151 & 383 & 597 \\
\hline & & $\mathrm{Aa}$ & $\mathrm{F}$ & 585 & 26 & 188 & 509 & 650 \\
\hline \multirow[t]{2}{*}{ Adour } & PROUZET, données non pulbliées & $\mathrm{Aa}$ & $M$ & 492 & 32 & 118 & 415 & 605 \\
\hline & & $\mathrm{Aa}$ & $\mathrm{F}$ & 539 & 25 & 101 & 475 & 600 \\
\hline \multirow[t]{2}{*}{ Lima } & ALEXANDRINO, 1996 & $\mathrm{Aa}$ & $M$ & 537 & 61 & 97 & 400 & 666 \\
\hline & & $\mathrm{Aa}$ & $\mathrm{F}$ & 634 & 44 & 86 & 480 & 733 \\
\hline \multirow[t]{2}{*}{ C. Bode } & ALEXANDRINO, 1996 & $\mathrm{Aa}$ & M & 421 & 20 & 12 & 375 & 450 \\
\hline & & $\mathrm{Aa}$ & $\mathrm{F}$ & 396 & 39 & 17 & 320 & 473 \\
\hline \multirow[t]{2}{*}{ Sebou } & SABATIE, 1993 & $\mathrm{Aa}$ & M & 549 & 4.4 & 482 & 445 & 670 \\
\hline & & $\mathrm{Aa}$ & $\mathrm{F}$ & 636 & 3.8 & 317 & 490 & 720 \\
\hline \multirow[t]{2}{*}{ Loire } & MENNESSON-BOISNEAU, 1990 & Af & $\mathrm{M}$ & 365 & 28 & 26 & 280 & 492 \\
\hline & & Af & $\mathrm{F}$ & 391 & 27 & 34 & 367 & 515 \\
\hline \multirow[t]{2}{*}{ Charente } & & Af & M & 376 & 29.2 & 5 & 340 & 415 \\
\hline & & Af & $\mathbf{F}$ & 438.2 & 27.3 & 28 & 405 & 510 \\
\hline \multirow[t]{2}{*}{ Gironde } & TAVERNY, 1991 & Af & $M$ & 383 & 27 & 34 & 320 & 450 \\
\hline & & Af & $\mathrm{F}$ & 437 & 27 & 38 & 392 & 509 \\
\hline \multirow[t]{2}{*}{ Lima } & ALEXANDRINO, 1993 & Af & $M$ & 391 & 47 & 51 & 284 & 468 \\
\hline & & Af & $\mathrm{F}$ & 421 & 38 & 54 & 342 & 560 \\
\hline \multirow[t]{2}{*}{ Rhône } & BAGLINIERE et al., 1996 & Af & $M$ & 465 & 47 & 81 & 363 & 556 \\
\hline & & Af & $\mathrm{F}$ & 533 & 33 & 341 & 415 & 657 \\
\hline \multirow[t]{2}{*}{ Sebou } & SABATIE, 1993 & Af & $M$ & 318 & 3 & 271 & 240 & 390 \\
\hline & & Af & $\mathrm{F}$ & 382 & 5 & 180 & 300 & 460 \\
\hline
\end{tabular}

Les aloses feintes de Charente ont des tailles moyennes relativement proches de celles de la Garonne (Tableau IX). Les plus gros individus se situent sur le Rhône ce qui pourrait confirmer la position taxinomique intermédiaire de cette sous-espèce Alosa fallax rhodanensis entre les deux espèces Alosa fallax et Alosa alosa.

Les résultats obtenus en terme d'âge des deux populations d'aloses de la Charente sont préliminaires et devront être critiqués en complétant les échantillons ultérieurement. En effet, le peu d'individus recueillis sur la Charente ne permettait pas de dresser une clef taille-âge pertinente. De plus, l'échantillon d'aloses feintes ne regroupait que $15 \%$ de 
mâles. Ce sex-ratio en faveur des femelles étant sans aucun doute lié aux mailles des filets maillant utilisés, trop larges $(60 \mathrm{~mm})$ pour capturer les plus petits mâles.

II faut noter que l'arbre phylogénétique construit ne peut être interprété qu'en tenant compte des cinq locus qui ont servi à sa construction. Aussi, au cours de l'avancement des travaux de caractérisation génétique et de la mise au point de nouveaux marqueurs pour les populations d'aloses, les arbres phylogénétiques seront modifiés. A chaque fois qu'un nouveau loci est découvert, les analyses doivent donc être reprises sur les individus caractérisés antérieurement. Or dans le cas de populations menacées ou éteintes cela est parfois impossible. II serait peut-être judicieux de standardiser une méthode de caractérisation génétique en désignant les locus les plus performants, ce qui permettrait d'uniformiser les recherches en Europe et de comparer ces résultats aux populations américaines. Une des raisons pour laquelle cette méthode de caractérisation génétique par électrophorèse n'a pas été standardisée est que la technique utilisant l'ADN mitochondrial, bien que non encore totalement maîtrisée en ce qui concerne les aloses, apparaît beaucoup plus prometteuse. La méthode retenue pour l'analyse de l'ADN mitochondrial est la PCR (Polymerase Chain Reaction). Actuellement, en ce qui concerne les aloses, les recherches sont dirigées vers l'identification d'endonucléases effectuant des coupures différentes selon l'espèce, le bassin d'origine et le degré d'hybridation des individus.

Les grandes aloses de la Charente apparaissent un peu plus polymorphes que les autres populations. Ce résultat est préliminaire car pour caractériser précisément les locus diagnostiques potentiels entre différentes populations il faudrait faire une AFC sur les matrices des fréquences alléliques observées chez ces populations. Néanmoins, les premiers résultats obtenus confirment la proximité génétique des populations de grandes aloses et donc du caractère très peu polymorphe avec les marqueurs protéiques de cette espèce, par rapport aux aloses feintes. Cela justifie la nécessité d'employer des techniques basées sur le polymorphisme de l'ADN pour détecter des différences potentielles plus subtiles entre les populations d'aloses. Les grandes aloses de la Charente, de l'Aulne et de la Garonne sont très proches génétiquement et se caractérisent par des niveaux de variabilité supérieurs de leurs polymorphismes si on les compare à ceux des populations de grandes aloses du Portugal étudiées, ce qui, en terme de homing, entre autre, est un phénomène intéressant à approfondir.

Il faut souligner que la population d'alose feinte de la Charente est la plus septentrionale caractérisée génétiquement à ce jour. Ceci pourrait expliquer en partie les différences de distribution des fréquences alléliques entre les moyennes de celles étudiées sur la façade Atlantique, celles du Rhône et celles de la Charente. La position des aloses feintes de la Charente sur l'arbre phylogénétique les rapproche des aloses feintes du nord du Portugal (Lima et Mondego), elles mêmes plus proches des aloses feintes du sud du Portugal que des aloses feintes du bassin méditerranéen (Rhône, Hérault, Aude) ou des aloses atlantiques méridionales (Oued Sebou) qui forment un groupe phylogénétique distinct.

\section{BIBLIOGRAPHIE}

ALEXANDRINO P., 1996. Estudo de populaçoes de savel (Alosa alosa L.) e savelha (Alosa fallax, Lacépède). Analise da diferenciaçao interspecifica, subestruturaçao e hibridaçao. Thèse Doctorat, Université de Porto, $180 \mathrm{p}$.

ALEXANDRINO P., BOISNEAU P., 2000. Diversité génétique In: Les aloses de l'Atlantique-est et de la Méditerrannée-ouest. Ecologie, biologie, taxinomie et influence des activités humaines. BAGLINIÈRE J.L. et ELIE P. (Eds.), INRA, Cemagref, Paris, 275 p. 
BAGLINIÈRE J.L, ELIE P., 2000. Les aloses de l'Atlantique-est et de la Méditerrannéeouest. Ecologie, biologie, taxinomie et influence des activités humaines. BAGLINIĖRE J.L. et ELIE P. (Eds.), INRA, Cemagref, Paris, 275 p.

BOISNEAU P., MENESSON-BOISNEAU C., GUYOMARD R., 1992. Electrophoretic identity between allis shad, Alosa alosa (L) and tacits shads, Alosa fallax (Lacépède). Journal of Fish Biology, 40, 731-738.

CASSOU LEINS F., CASSOU LEINS J.J., 1981. Recherche sur la biologie et l'halieutique des migrateurs de la Garonne et principalement de l'Alose. Thèse $3^{\text {ème }}$ cycle, Institut Polytechnique de Toulouse, $393 \mathrm{p}$.

LE CORRE M., 1999. Eco-biologie et génétique des populations d'Aloses (Alosa fallax spp.) de la Méditerranée française. Thèse doctorale de l'ENSA de Rennes, $173 \mathrm{p}$.

MENNESSON-BOISNEAU C., BOISNEAU P., 1990. Recherches sur les aloses du bassin de la Loire : Migration, Répartition, Reproduction, Caractéristiques biologiques et taxonomiques des aloses (Alosa sp.). Thèse doctorat (document de synthèse), Université de Rennes et de Paris Val de Marne, $143 \mathrm{p}$.

MENNESSON-BOISNEAU C., BAGLINIĖRE J.L., 1992. Mise au point d'une méthode de détermination de l'âge de la grande Alose (Alosa alosa) à partir des écailles. In: «Tissus durs et âge individuel des vertébrés». BAGLINIÈRE J.L., CASTANET J. , CONAND F., MEUNIER F. (Eds.), ORSTOM-INRA, Paris, 221-231.

PROUZET P., MARTINET J.P., BADIA J., 1994. Caractérisation biologique et variation des captures de grande Alose (Alosa alosa L.) par unité d'effort sur le fleuve Adour (Pyrénées Atlantiques, France). Aquatic Living Ressources, 7, 1-16.

ROCHARD E., 1992. Mise au point d'une méthode de suivi de l'abondance des amphihalins dans le système fluvio-estuarien de la Gironde, application à l'étude écobiologique de l'esturgeon Acipenser sturio. Cemagref, Thèse Doctorale de l'Univ. de Rennes I. 295 p.

SABATIÉ R., 1993. Recherches sur la biologie et l'écologie des aloses au Maroc (Alosa alosa Linné, 1758 et Alosa fallax Lacépède, 1803) : Exploitation et taxonomie des populations atlantiques, Bioécologie des aloses de l'Oued Sébou. Thèse doctorat, Université de Bretagne Occidentale, Brest, $326 \mathrm{p}$.

SABATIÉ M.R., BOISNEAU P., ALEXANDRINO P., 2000. Variabilité morphologique In: Les aloses de l'Atlantique-est et de la Méditerrannée-ouest. Ecologie, biologie, taxinomie et influence des activités humaines. BAGLINIĖRE J.L. et ELIE P. (Eds.), INRA, Cemagref, Paris, $275 \mathrm{p}$.

TAVERNY C., 1991. Contribution à la connaissance de la dynamique de populations d'aloses (Alosa alosa et Alosa fallax) dans le système fluvio-estuarien de la Gironde : Pêche, Biologie, Ecologie. Thèse Université de Bordeaux I, 451p.

VÉRON V., 1999. Les populations de grande Alose et d'Alose feinte des petits fleuves français du littoral Manche-Atlantique. Caractérisation morphologique, biologique et génétique. Mémoire de fin d'études pour l'obtention du DAA Halieutique. Laboratoires halieutique de I'ENSA de Rennes et INRA Ecologie aquatique de Rennes. 81 p. 
